\title{
Shape Optimization in Unsteady Blood Flow: A Numerical Study of Non-Newtonian Effects
}

\author{
FEBY ABRAHAM \\ Mechanical Engineering and Materials Science, Rice University - MS 321, 6100 Main Street, Houston, TX 77005, USA \\ MAREK BEHR \\ Lehrstuhl für Numerische Mechanik, TU München, Boltzmannstr. 15, 85747 Garching, Germany \\ MATTHIAS HEINKENSCHLOSS \\ Computational and Applied Mathematics, Rice University - MS 134, 6100 Main Street, Houston, TX 77005, USA \\ August 11, 2004

\begin{abstract}
This paper presents a numerical study of non-Newtonian effects on the solution of shape optimization problems involving unsteady pulsatile blood flow. We consider an idealized twodimensional arterial graft geometry. Our computations are based on the Navier-Stokes equations generalized to non-Newtonian fluid, with the Carreau-Yasuda model employed to account for the shear-thinning behavior of blood. Using a gradient-based optimization algorithm, we compare the optimal shapes obtained using both the Newtonian and generalized Newtonian constitutive equations. Depending on the shear rate prevalent in the domain, substantial differences in the flow as well as in the computed optimal shape are observed when the Newtonian constitutive equation is replaced by the Carreau-Yasuda model. By varying a geometric parameter in our test case, we investigate the influence of the shear rate on the solution.
\end{abstract}

\section{Introduction}

There has been growing interest in the use of Computational Fluid Dynamics (CFD) in the design of biomedical devices involving blood flow. Example applications studied previously include the design of axial-flow blood pumps [1,2], the design of prosthetic grafts [3,4], among others. Typically, the flow exhibits strong unsteady behavior, and therefore requires the use of the time-dependent form of the governing equations of incompressible flow. In addition, the viscoelastic and shear-thinning behavior of blood needs to be taken into account [5]. The shear-thinning effect is usually handled by employing a generalized Newtonian constitutive equation. Gijsen et al. [6] and more recently Neofytou et al. [7] have shown significant differences in the flow field, when the shear-thinning behavior of blood is accounted for.

Prior research on optimal design involving blood flow has mostly been performed in the context of a steady approximation of the governing equations, or using a Newtonian constitutive model (e.g., $[1-4,8]$ ). The focus of the current paper is to investigate the effect of shear-thinning behavior of blood on the solution of optimal design problems in unsteady blood flow. In the earlier report [4], we studied the effect of the constitutive equation choice on the solution of the optimal design problem in the context of steady blood flows. In that paper, we saw that the constitutive equation effect on the solution of the optimal shape design problem depends on the shear rate in the domain. The present paper extends the investigation in [4] to unsteady blood flow.

With the long-term goal of solving large-scale shape optimization problems for unsteady flows, potentially involving moving boundaries, the present numerical study is a step in the direction of 
addressing the issues that arise in the optimal design of centrifugal blood pumps [9]. Blood flow in these pumps is simulated using the Deformable-Spatial-Domain/Stabilized-Space-Time (DSD/SST) finite element formulation [10,11], as well as the Shear-Slip Mesh Update Method (SSMUM) [12,13], which accounts for the mesh deformation due to the rotating impeller. A shape optimization problem in this context could involve finding the shape of pump components based on a certain parameterization that minimizes hemolysis.

The governing equations for the fluid flow and a model shape optimization problem are described in Section 2. For the numerical solution of the shape optimization problem we discretize the NavierStokes equation using Galerkin/Least-Squares (GLS) stabilized piecewise-linear finite elements. The discretized shape optimization problem is solved using a gradient-based optimization algorithm. The details of the finite element discretization of the problem, computation of derivatives in our optimization problem as well as specifications of the optimization algorithm used are given in Section 3. The numerical results for our test case are reported in Section 4.

\section{A model shape optimization problem}

We first introduce the equations for blood flow in a fixed domain $\Omega \subset \mathbb{R}^{n_{\text {sd }}}$, where $n_{\text {sd }}$ is the number of space dimensions. The symbols $\mathbf{u}$ and $p$ represent the velocity and pressure. The boundary $\partial \Omega$ of $\Omega$ is decomposed into two disjoint segments $\Gamma_{h}$ and $\Gamma_{g}$. The momentum and mass balance equations subject to Neumann and Dirichlet boundary conditions, can be written as

$$
\begin{array}{rlll}
\rho\left(\frac{\partial \mathbf{u}}{\partial t}+\mathbf{u} \cdot \nabla \mathbf{u}\right)-\boldsymbol{\nabla} \cdot \boldsymbol{\sigma}(\mathbf{u}, p) & =\mathbf{0} & \text { on } \Omega, \\
\boldsymbol{\nabla} \cdot \mathbf{u} & =\mathbf{0} & \text { on } \Omega, \\
\mathbf{n} \cdot \boldsymbol{\sigma}(\mathbf{u}, p) & =\boldsymbol{h} & \text { on } \Gamma_{\boldsymbol{h}}, \\
\mathbf{u} & =\boldsymbol{g} & & \text { on } \Gamma_{\boldsymbol{g}} .
\end{array}
$$

The stress tensor $\boldsymbol{\sigma}$ is given by

$$
\boldsymbol{\sigma}(\mathbf{u}, p)=-p \mathbf{I}+2 \mu \varepsilon(\mathbf{u}), \quad \varepsilon(\mathbf{u})=\frac{1}{2}\left(\nabla \mathbf{u}+\nabla \mathbf{u}^{T}\right),
$$

where $\mu$ is the dynamic viscosity, $\rho$ is the density and $\mathbf{I}$ denotes the identity tensor. For a Newtonian fluid $\mu$ is a constant, whereas in the case of a generalized Newtonian fluid, we incorporate the shearthinning behavior of blood, as expressed by the Carreau-Yasuda model:

$$
\mu(\dot{\gamma})=\mu_{\infty}+\frac{\mu_{0}-\mu_{\infty}}{\left(1+(\lambda \dot{\gamma})^{b}\right)^{a}}
$$

Here, $\mu_{\infty}$ and $\mu_{0}$ are the infinite shear viscosity and the zero shear viscosity, respectively. The parameter values follow [14]: $\mu_{\infty}=0.0035 \mathrm{~Pa} \mathrm{~s}, \mu_{0}=0.1600 \mathrm{~Pa} \mathrm{~s}, \lambda=8.2 \mathrm{~s}, a=1.23$ and $b=0.64$. For parallel shear flows, the shear rate is given by

$$
\dot{\gamma}=\sqrt{2 \varepsilon(\mathbf{u}): \varepsilon(\mathbf{u})}
$$

The fluid density is taken as $\rho=1.058 \mathrm{~g} / \mathrm{cm}^{3}$.

For the differential equation described in (1), a weak form is constructed as follows. We define the function spaces

$$
\begin{aligned}
& \mathcal{S}=\left\{\mathbf{u} \mid \mathbf{u} \in\left[H^{1}(\Omega)\right]^{n_{\mathrm{sd}}}, \mathbf{u}=\boldsymbol{g} \text { on } \Gamma_{\boldsymbol{g}}\right\}, \\
& \mathcal{V}=\left\{\mathbf{u} \mid \mathbf{u} \in\left[H^{1}(\Omega)\right]^{n_{\mathrm{sd}}}, \mathbf{u}=\mathbf{0} \quad \text { on } \Gamma_{\boldsymbol{g}}\right\},
\end{aligned}
$$


where $H^{1}(\Omega)$ is defined in the usual way $[15,16]$. The weak form of (1) is to find $\mathbf{u} \in \mathcal{S}$ and $p \in L^{2}(\Omega)$, such that

$$
\int_{\Omega} \mathbf{w} \cdot \rho\left(\frac{\partial \mathbf{u}}{\partial t}+\mathbf{u} \cdot \nabla \mathbf{u}\right) d \mathbf{x}+\int_{\Omega} \varepsilon(\mathbf{w}): \boldsymbol{\sigma}(\mathbf{u}, p) d \mathbf{x}+\int_{\Omega} q \boldsymbol{\nabla} \cdot \mathbf{u} d \mathbf{x}=\int_{\Gamma_{h}} \mathbf{w} \cdot \boldsymbol{h} d \mathbf{x}
$$

for all $\mathbf{w} \in \mathcal{V}$ and for all $q \in L^{2}(\Omega)$.

Our goal is to find a shape $\Omega$, such that a given objective function $J$, which depends on $\mathbf{u}(\mathbf{x}, t), p(\mathbf{x}, t)$ and on $\Omega$ itself, is minimized. We consider the case where the set of admissible shapes can be parameterized by $\boldsymbol{\alpha} \in \mathcal{A}_{a d} \subset \mathbb{R}^{n}$. The optimal shape design problem is given as follows:

$$
\begin{aligned}
\operatorname{minimize} & \hat{J}(\boldsymbol{\alpha}), \\
\text { subject to } & \boldsymbol{\alpha} \in \mathcal{A}_{a d} .
\end{aligned}
$$

where $\hat{J}(\boldsymbol{\alpha})=J(\mathbf{u}(\boldsymbol{\alpha}), p(\boldsymbol{\alpha}), \boldsymbol{\alpha})$ and $(\mathbf{u}(\boldsymbol{\alpha}), p(\boldsymbol{\alpha}))$ is the solution of $(5)$ with $\Omega=\Omega(\boldsymbol{\alpha})$. We consider the shear rate integral, considered in $[4,8]$, as our objective function, i.e.,

$$
J(\mathbf{u}, p, \boldsymbol{\alpha})=\int_{t_{L}}^{t_{U}} \Phi(\mathbf{u}, \boldsymbol{\alpha}),
$$

a scalar metric, where $\Phi(\mathbf{u}, \boldsymbol{\alpha})=2 \int_{\Omega_{\mathrm{obs}}(\boldsymbol{\alpha})} \varepsilon(\mathbf{u}): \varepsilon(\mathbf{u}) d \mathbf{x}$, with $\Omega_{\text {obs }}(\boldsymbol{\alpha}) \subset \Omega(\boldsymbol{\alpha})$, representing the observation region, and $t_{U}$ corresponding to the final time $t_{U}=T$. We also refer to (7) as the dissipation function. The shear rate integral is a critical representative measure for the damage to red blood cells (hemolysis), platelet aggregation, and thrombus formation on artificial surfaces. More useful, yet sophisticated objective functions are possible, such as the tensor-based measure for estimating blood damage introduced by Arora et al. [17], where blood cells are modeled as deforming droplets and their deformation is tracked along the pathlines of the computed flow. However (7) is a reasonable measure for the purpose of this paper.

\section{Numerical solution of the optimization problem}

\subsection{Discretization of the optimization problem}

To discretize the governing equations (5), we apply a stabilized finite element discretization using conforming piecewise linear finite elements for the velocities and the pressure. Let $\left\{\Omega^{e}(\boldsymbol{\alpha}) \mid e=\right.$ $\left.1,2, \ldots, n_{\mathrm{el}}\right\}$ be a triangulation of $\Omega(\boldsymbol{\alpha})$. We set

$$
\begin{aligned}
& H^{1 h}(\Omega(\boldsymbol{\alpha}))=\left\{v \in C(\Omega(\boldsymbol{\alpha})) \mid v \text { is linear on } \Omega^{e}(\boldsymbol{\alpha}), e=1,2, \ldots, n_{\mathrm{el}}\right\}, \\
& \mathcal{S}^{h}=\left\{\mathbf{u}^{h} \mid \mathbf{u}^{h} \in\left[H^{1 h}(\Omega(\boldsymbol{\alpha}))\right]^{n_{\mathrm{sd}}}, \mathbf{u}^{h}=\boldsymbol{g}^{h} \quad \text { on } \Gamma_{\boldsymbol{g}}(\boldsymbol{\alpha})\right\}, \\
& \mathcal{V}^{h}=\left\{\mathbf{u}^{h} \mid \mathbf{u}^{h} \in\left[H^{1 h}(\Omega(\boldsymbol{\alpha}))\right]^{n_{\mathrm{sd}}}, \mathbf{u}^{h}=\mathbf{0} \quad \text { on } \Gamma_{\boldsymbol{g}}(\boldsymbol{\alpha})\right\} .
\end{aligned}
$$

The GLS-stabilized finite element discretization of (5) is given as follows. Find $\mathbf{u}^{h} \in \mathcal{S}^{h}$ and $p^{h} \in H^{1 h}(\Omega(\boldsymbol{\alpha}))$, such that

$$
\begin{aligned}
& \int_{\Omega(\boldsymbol{\alpha})} \mathbf{w}^{h} \cdot \rho\left(\frac{\partial \mathbf{u}^{h}}{\partial t}+\mathbf{u}^{h} \cdot \nabla \mathbf{u}^{h}\right) d \mathbf{x}^{h}+\int_{\Omega(\boldsymbol{\alpha})} \boldsymbol{\varepsilon}\left(\mathbf{w}^{h}\right): \boldsymbol{\sigma}\left(\mathbf{u}^{h}, p^{h}\right) d \mathbf{x}^{h} \\
& \quad+\sum_{e=1}^{n_{\mathrm{el}}} \int_{\Omega^{e}(\boldsymbol{\alpha})} \frac{\tau_{\mathrm{MOM}}}{\rho}\left[\rho\left(\frac{\partial \mathbf{w}^{h}}{\partial t}+\mathbf{u}^{h} \cdot \nabla \mathbf{w}^{h}\right)-\boldsymbol{\nabla} \cdot \boldsymbol{\sigma}\left(\mathbf{w}^{h}, q^{h}\right)\right] \cdot\left[\rho\left(\frac{\partial \mathbf{u}^{h}}{\partial t}+\mathbf{u}^{h} \cdot \nabla \mathbf{u}^{h}\right)-\boldsymbol{\nabla} \cdot \boldsymbol{\sigma}\left(\mathbf{u}^{h}, p^{h}\right)\right] d \mathbf{x}^{h} \\
& \quad+\int_{\Omega(\boldsymbol{\alpha})} q^{h} \boldsymbol{\nabla} \cdot \mathbf{u}^{h} d \mathbf{x}^{h}=\int_{\Gamma_{h}(\boldsymbol{\alpha})} \mathbf{w}^{h} \cdot \boldsymbol{h}^{h} d \mathbf{x}^{h}
\end{aligned}
$$


for all $\mathbf{u}^{h} \in \mathcal{V}^{h}$ and for all $q^{h} \in H^{1 h}(\Omega(\boldsymbol{\alpha}))$. The GLS stabilization [10,18,19] provides stability at high element-level Reynolds numbers and circumvents the compatibility conditions restricting the choice of interpolation function spaces. In particular, it allows the use of equal-order piecewise linear functions to approximate both the velocity and the pressure. The coefficient for the GLS stabilization, $\tau_{\mathrm{MOM}}$, in (8) is defined, e.g., in [19]; it depends on the element length $h_{e}$, which in our computations is specified by the adv definition in [20].

Our choice of piecewise linear functions makes our discrete approximation a low-order method, i.e. the order of the function space is lower than the order of the highest derivative in (1), the stabilized finite element equivalent of which is (8). Therefore, the terms $\boldsymbol{\nabla} \cdot \boldsymbol{\sigma}\left(\mathbf{v}^{h}, q^{h}\right)$ and $\boldsymbol{\nabla}$. $\boldsymbol{\sigma}\left(\mathbf{u}^{h}, p^{h}\right)$ in (8) are given by $\boldsymbol{\nabla} q^{h}$ and $\boldsymbol{\nabla} p^{h}$, respectively. Consequently, our stabilized finite element formulation is only "weakly consistent". A variational reconstruction of second derivatives [21,22] could be used to improve the consistency of our approximation.

We use the generalized midpoint rule for the time-discretization of $\mathbf{u}^{h}$, i.e., we approximate $\partial \mathbf{u}^{h} / \partial t$ and $\mathbf{u}^{h}$ in (8) by

$$
\mathbf{u}^{h}=\gamma \mathbf{u}_{i}+(1-\gamma) \mathbf{u}_{i+1}, \quad \frac{\partial \mathbf{u}^{h}}{\partial t}=\frac{\mathbf{u}_{i+1}-\mathbf{u}_{i}}{\Delta t}
$$

where $0 \leq \gamma \leq 1$, and $\mathbf{u}_{i}$ represents the discrete velocity field in the domain at time step $i$. The time derivative of the velocity weighting function, $\partial \mathbf{w}^{h} / \partial t$, represents the variation of time-derivative of the velocity. In the case of the generalized midpoint rule with time-step $\Delta t(9)$ with $\mathbf{u}_{i}$ known, this term becomes simply $\mathbf{w}^{h} / \Delta t$.

Let $\mathbf{x}^{h}(\boldsymbol{\alpha}) \in \Omega(\boldsymbol{\alpha})$ denote the nodes associated with the nodal basis for $H^{1 h}(\Omega(\boldsymbol{\alpha}))$. For the $i$ th time step, we can represent (8)-(9), symbolically as:

$$
\mathbf{c}_{i}\left(\mathbf{v}_{i+1}(\boldsymbol{\alpha}), \mathbf{v}_{i}(\boldsymbol{\alpha}), \mathbf{x}^{h}(\boldsymbol{\alpha})\right)=\mathbf{0}, \quad i=0, \ldots, L, \ldots, U-1,
$$

where $\mathbf{v}_{i}(\boldsymbol{\alpha})=\left(\mathbf{u}_{i}(\boldsymbol{\alpha}), p_{i}(\boldsymbol{\alpha})\right)$ is the solution of (8), (9) at time step $i$ and time steps $0, L$ and $U$ correspond to $t_{0}, t_{L}$ and $t_{U}$, respectively.

We assume that for any $\boldsymbol{\alpha} \in \mathcal{A}_{a d}$, the equation (10) has a unique solution $\mathbf{v}_{i}(\boldsymbol{\alpha}), \quad i=1, \ldots, U$. We compute an approximation of this solution using a Newton GMRES method [23].

Remark 3.1 The second term in (8) contains $\int_{\Omega(\boldsymbol{\alpha})} \varepsilon\left(\mathbf{w}^{h}\right): 2 \mu \varepsilon\left(\mathbf{u}^{h}\right) d \mathbf{x}^{h}$. When the CarreauYasuda model is used, $\mu$ depends on $\mathbf{u}\left(c f\right.$. (3)-(4)). In this case, the evaluation of $\frac{\partial \mathbf{c}_{i}}{\partial \mathbf{u}^{h}}$ requires us to compute the derivative of $\mu$ with respect to $\mathbf{u}^{h}$. In our computations we drop this derivative, which leads to an inexactness in the Jacobian $\frac{\partial \mathbf{c}_{i}}{\partial \mathbf{u}^{h}}$. While this inexact Jacobian may adversely affect the convergence of the Newton GMRES method, we have found the performance of our Newton GMRES method satisfactory, and we choose therefore to avoid the additional implementation effort required to compute the exact Jacobian $\frac{\partial \mathbf{c}_{i}}{\partial \mathbf{u}^{h}}$.

The discretized shape optimization problem may now be written as:

$$
\begin{aligned}
\operatorname{minimize} & \widehat{J}^{h}(\boldsymbol{\alpha}), \\
\text { subject to } & \boldsymbol{\alpha} \in \mathcal{A}_{a d},
\end{aligned}
$$

where

$$
\begin{aligned}
\widehat{J}^{h}(\boldsymbol{\alpha}) & =J^{h}\left(\mathbf{v}_{L}(\boldsymbol{\alpha}), \ldots, \mathbf{v}_{U}(\boldsymbol{\alpha}), \mathbf{x}^{h}(\boldsymbol{\alpha}), \boldsymbol{\alpha}\right) \\
& =\frac{\Delta t}{2} \sum_{i=L}^{U-1}\left[\Phi^{h}\left(\mathbf{u}_{i}(\boldsymbol{\alpha}), \mathbf{x}^{h}(\boldsymbol{\alpha})\right)+\Phi^{h}\left(\mathbf{u}_{i+1}(\boldsymbol{\alpha}), \mathbf{x}^{h}(\boldsymbol{\alpha})\right)\right]
\end{aligned}
$$




\subsection{Gradient computation}

We use a gradient-based algorithm to solve (11). The design variables $\boldsymbol{\alpha}$ enter (11) implicitly accounting for the dependence of the state field $\mathbf{v}_{i}, i=0, \ldots, L, \ldots, U$, as well as the nodal coordinates $\mathbf{x}^{h}$, on $\boldsymbol{\alpha}$, and explicitly, whenever regularization terms are included in the objective function. Using the implicit function theorem on (10), we obtain the first-order discrete sensitivity equations as:

$$
\frac{\partial \mathbf{c}_{i}}{\partial \mathbf{v}_{i+1}} \frac{d \mathbf{v}_{i+1}}{d \boldsymbol{\alpha}}=-\frac{\partial \mathbf{c}_{i}}{\partial \mathbf{v}_{i}} \frac{d \mathbf{v}_{i}}{d \boldsymbol{\alpha}}-\frac{\partial \mathbf{c}_{i}}{\partial \mathbf{x}^{h}} \frac{d \mathbf{x}^{h}}{d \boldsymbol{\alpha}}, \quad i=0, \ldots, L, \ldots, U-1 .
$$

Let $\Phi_{i+1}=\Phi^{h}\left(\mathbf{u}_{i+1}(\boldsymbol{\alpha}), \mathbf{x}^{h}(\boldsymbol{\alpha})\right)$; then the sensitivity $d \Phi_{i+1} / d \boldsymbol{\alpha}$ at time $t_{i+1}$ is:

$$
\frac{d \Phi_{i+1}}{d \boldsymbol{\alpha}}=\frac{\partial \Phi_{i+1}}{\partial \mathbf{x}^{h}} \frac{d \mathbf{x}^{h}}{d \boldsymbol{\alpha}}+\frac{\partial \Phi_{i+1}}{\partial \mathbf{v}_{i+1}} \frac{d \mathbf{v}_{i+1}}{d \boldsymbol{\alpha}}, \quad i=0, \ldots, L, \ldots, U-1
$$

Finally, the gradient $\nabla \widehat{J}^{h}$ of (12) is given as

$$
\nabla \widehat{J}^{h}=\frac{\partial J^{h}}{\partial \boldsymbol{\alpha}}+\frac{\Delta t}{2} \sum_{i=L}^{U-1}\left[\frac{d \Phi_{i}}{d \boldsymbol{\alpha}}+\frac{d \Phi_{i+1}}{d \boldsymbol{\alpha}}\right] .
$$

The dependence of our geometry on $\boldsymbol{\alpha}$ is identical to that in [22], and consequently, the term $\frac{d \mathrm{x}^{h}}{d \boldsymbol{\alpha}}$ is computed as described in [22]. The recipe for the computation of the term $\frac{\partial \mathbf{c}_{i}}{\partial \mathbf{x}^{h}} \frac{d \mathbf{x}^{h}}{d \boldsymbol{\alpha}}$ is shown in Appendix $\mathrm{C}$ of $[22]$.

If the number of our design variables is large, the gradient (15) can also be evaluated using the adjoint equation method. The Lagrangian associated with the discretized optimization problem is given by

$$
\begin{aligned}
& L\left(\mathbf{v}_{0}, \ldots, \mathbf{v}_{U}, \mathbf{z}_{1}, \ldots, \mathbf{z}_{U}, \boldsymbol{\alpha}\right) \\
& \quad=J^{h}\left(\mathbf{v}_{L}, \ldots, \mathbf{v}_{U}, \mathbf{x}^{h}(\boldsymbol{\alpha}), \boldsymbol{\alpha}\right)+\sum_{i=0}^{U-1} \mathbf{z}_{i+1}^{T} \mathbf{c}_{i}\left(\mathbf{v}_{i+1}, \mathbf{v}_{i}, \mathbf{x}^{h}(\boldsymbol{\alpha})\right) \\
& \quad=\frac{\Delta t}{2} \sum_{i=L}^{U-1}\left[\Phi_{i}+\Phi_{i+1}\right]+\sum_{i=0}^{U-1} \mathbf{z}_{i+1}^{T} \mathbf{c}_{i}\left(\mathbf{v}_{i+1}, \mathbf{v}_{i}, \mathbf{x}^{h}(\boldsymbol{\alpha})\right) .
\end{aligned}
$$

where $\mathbf{z}_{i}$ represent the adjoint variables $\mathbf{z}^{h}=\left(\boldsymbol{\lambda}^{h}, \theta^{h}\right)$ at time step $i$. Notice that in (16), we have not written the dependence of the state $\mathbf{v}_{i}$ on $\boldsymbol{\alpha}$ explicitly. This is because we are explicitly accounting for the state equation. We only write $\mathbf{v}_{i}(\boldsymbol{\alpha})$ when using the modified objective function (11). The adjoint equation is then obtained by setting $\frac{\partial L}{\partial \mathbf{v}_{i}}=\mathbf{0}$, for $i=1, \ldots, U$. This yields:

$$
\begin{aligned}
\left(\frac{\partial \mathbf{c}_{U-1}\left(\mathbf{v}_{U}, \mathbf{v}_{U-1}, \mathbf{x}^{h}(\boldsymbol{\alpha})\right)}{\partial \mathbf{v}_{U}}\right)^{T} \mathbf{z}_{U}= & -\frac{\Delta t}{2}\left(\frac{\partial \Phi_{U}}{\partial \mathbf{v}_{U}}\right)^{T} \\
\left(\frac{\partial \mathbf{c}_{i-1}\left(\mathbf{v}_{i}, \mathbf{v}_{i-1}, \mathbf{x}^{h}(\boldsymbol{\alpha})\right)}{\partial \mathbf{v}_{i}}\right)^{T} \mathbf{z}_{i}= & -\left(\frac{\partial \mathbf{c}_{i}\left(\mathbf{v}_{i+1}, \mathbf{v}_{i}, \mathbf{x}^{h}(\boldsymbol{\alpha})\right)}{\partial \mathbf{v}_{i}}\right)^{T} \mathbf{z}_{i+1}-\chi_{i} \Delta t\left(\frac{\partial \Phi_{i}}{\partial \mathbf{v}_{i}}\right)^{T} \\
& i=U-1, U-2, \ldots, 1,
\end{aligned}
$$

where $\chi_{i}$ is defined as

$$
\chi_{i}= \begin{cases}0 & \text { for } 0 \leq i<L \\ 0.5 & \text { for } i=L \\ 1 & \text { for } L<i<U-1\end{cases}
$$


The gradient is then obtained as follows:

$$
\frac{\partial L}{\partial \boldsymbol{\alpha}}=\frac{\partial J^{h}}{\partial \boldsymbol{\alpha}}+\frac{\Delta t}{2} \sum_{i=L}^{U-1}\left[\frac{\partial \Phi_{i}}{\partial \mathbf{x}^{h}}+\frac{\partial \Phi_{i+1}}{\partial \mathbf{x}^{h}}\right] \frac{d \mathbf{x}^{h}}{d \boldsymbol{\alpha}}+\Delta t \sum_{i=0}^{U-1} \mathbf{z}_{i+1}^{T} \frac{\partial \mathbf{c}_{i}\left(\mathbf{v}_{i+1}, \mathbf{v}_{i}, \mathbf{x}^{h}(\boldsymbol{\alpha})\right)}{\partial \mathbf{x}^{h}} \frac{d \mathbf{x}^{h}}{d \boldsymbol{\alpha}}
$$

Remark 3.2 When using the Carreau-Yasuda model (3)-(4), the viscosity $\mu$ depends on $\mathbf{u}^{h}(\boldsymbol{\alpha})$ and on $\mathbf{x}^{h}(\boldsymbol{\alpha})$. (The dependence on $\mathbf{u}^{h}(\boldsymbol{\alpha})$ is obvious, the dependence on $\mathbf{x}^{h}(\boldsymbol{\alpha})$ is due to the fact that the Jacobians of $\mathbf{u}^{h}(\boldsymbol{\alpha})$ are required (see, e.g., [24] or [25]).) We have already mentioned in Remark 3.1 that in our computations, the terms involving partial derivatives $\frac{\partial \dot{\gamma}}{\partial \mathbf{u}^{h}}$ are dropped from the computation of $\frac{\partial \mathbf{c}^{h}}{\partial \mathbf{u}^{h}}$. We also drop terms involving partial derivatives $\frac{\partial \dot{\gamma}}{\partial \mathbf{x}^{h}}$ from the computation of $\frac{\partial \mathbf{c}^{h}}{\partial \mathbf{x}^{h}}$. Therefore our computed gradient $\nabla \widehat{J}^{h}(\boldsymbol{\alpha})$ is inexact when using the Carreau-Yasuda constitutive model.

Note that we follow the discretize-then-optimize approach, i.e., we first discretize the optimization problem to obtain (11) and then we solve the resulting nonlinear programming problem (6). Other approaches are possible. We refer to [22] for more details.

\subsection{Optimization algorithm}

The conceptual algorithm for the solution of the shape optimization problem is then

1. Initialize design variable $\boldsymbol{\alpha}$ and generate $\mathbf{x}^{h}(\boldsymbol{\alpha})$.

2. Set the initial conditions on the velocity $\mathbf{u}^{0}$ and the sensitivity $\frac{d \mathbf{u}_{0}}{d \boldsymbol{\alpha}}$.

3. While not converged:

(a) solve mesh sensitivity equation to obtain $\frac{d \mathbf{x}^{h}}{d \boldsymbol{\alpha}}$.

(b) for each time step $i=1, \ldots, U$ :

i. solve for $\mathbf{v}_{i+1}(\boldsymbol{\alpha})$ using (10).

ii. if sensitivity approach; solve for $\frac{d \mathbf{v}_{i+1}(\boldsymbol{\alpha})}{d \boldsymbol{\alpha}}$ using (13), accumulate objective function $\widehat{J}$ if necessary using (12), and accumulate $\nabla \widehat{J}(\boldsymbol{\alpha})$ using (14) and (15).

(c) if adjoint approach, solve for $\mathbf{z}$ and $\nabla \widehat{J}^{h}(\boldsymbol{\alpha})$ using (17) and (19),

(d) use $\nabla \widehat{J}^{h}$ to determine design update $\delta \boldsymbol{\alpha}$ and set $\boldsymbol{\alpha}=\boldsymbol{\alpha}+\delta \boldsymbol{\alpha}$.

(e) adapt the mesh to obtain $\mathbf{x}^{h}(\boldsymbol{\alpha})$.

We use a BFGS quasi-Newton method to compute the design update $\delta \boldsymbol{\alpha}$ in step (d).

\section{Arterial grafting}

As a test case, we consider the arterial grafting problem. Figure 1 shows the geometry of the problem, with the flow proceeding from left to right. The boundary conditions for the modeled flow field are: specified pulsatile inlet velocity, no-slip boundary conditions at all solid walls including the graft, and a parallel flow condition at the outlet. The geometry of the artery (rectangles in Figure 1) is fixed. The shape of the graft is optimized. We use the parameterization

$$
r(\theta)=\sum_{i=0}^{P} r_{i} \theta^{i}
$$


with $P=5$ to represent the centerline of the graft.

Two conditions that ensure that location of the connection between graft and artery is fixed are used to express $r_{0}$ and $r_{1}$ in terms of the other parameters. Hence, our design variables $\boldsymbol{\alpha}$ consist of $r_{i}, i=2, \ldots, P$. Let $H=0.8$ be the height at the inlet.

The mesh in the subdomain representing the artery is fixed. For a given centerline (20), the nodal points $\mathbf{x}^{h}$ of the triangulation of the graft are obtained by moving in the radial direction from the centerline by a constant distance. This defines the map $\boldsymbol{\alpha} \mapsto \mathbf{x}^{h}(\boldsymbol{\alpha})$.

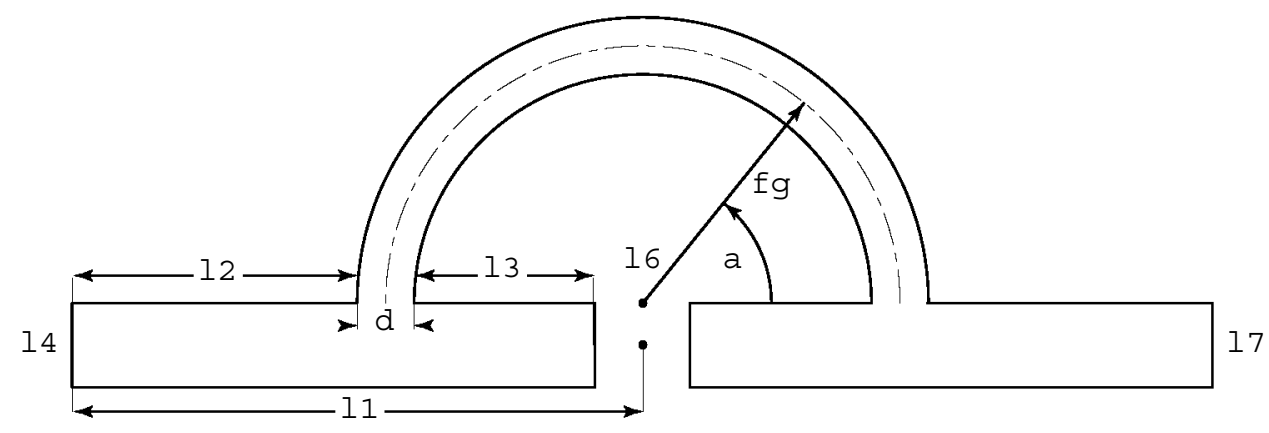

Figure 1. Computational domain for the arterial graft. The initial shape of the design curve of the graft (dashed line) is a semi-circle with center at $C$. Fixed geometry parameters are $l_{1}=6.0$, $l_{2}=3.0, l_{3}+d=2.5$. The downstream section of the host artery is symmetric with respect to the upstream one.

The inflow velocity profile is given as:

$$
u_{x}=q(t) V(y), \quad u_{y}=0 .
$$

he term $V(y)$ represents the parabolic spatial dependence of the velocity profile at the inlet with maximum velocity $V_{\max }$ :

$$
V(y)=\frac{4 V_{\max }}{H^{2}} y(H-y),
$$

where $y=0$ represents the bottom-wall of the artery. The temporal flow modulation is given by:

$$
q(t)= \begin{cases}0.1 t & \text { for } 0 \leq t<5 \\ 0.5 & \text { for } 5 \leq t<8 \\ 0.5+0.1 \sin [\omega(t-8)] & \text { for } 8 \leq t<\infty\end{cases}
$$

with $\omega=2 \pi$. We define Reynolds number as $R e=\rho V_{\max } H / \mu_{\infty}$, and set $V_{\max }$ so that $R e=300$. We define the Womersley number as Wo $=\frac{H}{2} \sqrt{\frac{\omega \rho}{\mu_{\infty}}}$, which is 5.51 for the flow in (23). The Womersley number defines the ratio of inertial forces to viscous forces; a study on Womersley number effects in blood flow in arteries can be found in [26,27]. Our numerical results make use of the the semidiscrete finite element formulation using the sensitivity-based approach to compute gradients. In all computations, the viscosity for the Newtonian case is chosen to be $\mu=\mu_{\infty}$. The observation region $\Omega_{\text {obs }}$ for both the test cases is the entire flow domain, and $t_{L}=15$ and $t_{U}=20$. Just as in the steady case [4], we have observed little impact of the constitutive model on the optimal shape when $R e=50$. Therefore, we do not show the $R e=50$ results here. 


\subsection{Case 1}

We define the aspect ratio as the ratio of the diameter $d$ to height $H$. The first case involves $d=0.6$ resulting in an aspect ratio of 0.75 . The optimization process leads to a $10.98 \%$ reduction in the dissipation function using the Newtonian constitutive equation, with a gradient norm of $\left\|\nabla \widehat{J}\left(\boldsymbol{\alpha}_{0}\right)\right\| \times 10^{-3}$, and a $9.30 \%$ reduction in the dissipation function using the Carreau-Yasuda model, with a gradient norm of $\left\|\nabla \widehat{J}\left(\boldsymbol{\alpha}_{0}\right)\right\| \times 10^{-3}$. Figures 2 and 3 show the variation of $\Phi\left(\mathbf{u}^{h}\right)$ as a function of time for the initial and optimal shapes, using the Newtonian and Carreau-Yasuda constitutive equations, respectively. Figure 4 illustrates that the optimal shapes obtained by the different constitutive equation choices do not differ significantly in this case. Table 1 shows the computed optimal shape parameters defined in (20), and Table 2 shows the values of the angles of the graft (from horizontal) at the inlet and exit of the artery. Figures 5 and 6 show the streamline plots for the initial and optimal shapes, respectively, using the Newtonian constitutive equation within one period of the pulsatile phase of the flow. Figures 7 and 8 show the streamline plots for the initial and optimal shapes, respectively, using the Carreau-Yasuda constitutive equation within one period of the pulsatile phase of the flow.

\begin{tabular}{lrrr}
\hline \hline Design Variable & Initial & Newtonian & Carreau-Yasuda \\
\hline$r_{2}$ & 0.0 & 1.025 & 0.7142 \\
$r_{3}$ & 0.0 & -0.518 & -0.2720 \\
$r_{4}$ & 0.0 & 0.1460 & 0.0706 \\
$r_{5}$ & 0.0 & -0.0136 & -0.0057 \\
\hline \hline
\end{tabular}

Table 1. The design variables for the graft optimization for case 1.

\begin{tabular}{llrr}
\hline \hline Angle & Initial & Newtonian & Carreau-Yasuda \\
\hline$\theta=0$ & $90.00^{\circ}$ & $64.21^{\circ}$ & $66.18^{\circ}$ \\
$\theta=\pi$ & $90.00^{\circ}$ & $65.05^{\circ}$ & $65.80^{\circ}$ \\
\hline \hline
\end{tabular}

Table 2. The angle that the graft makes with the artery at its inlet and exit, for case 1 .
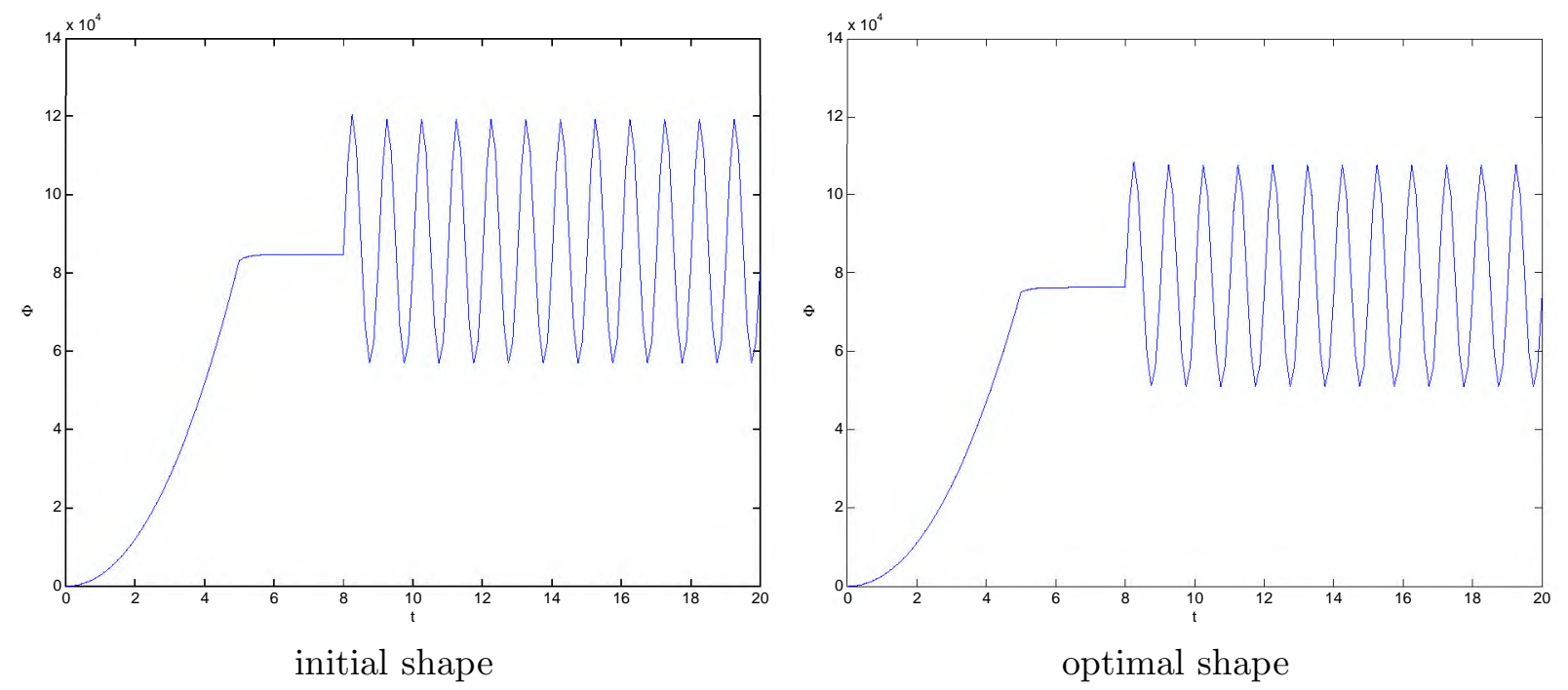

Figure 2. The function $\Phi\left(\mathbf{u}^{h}\right)$ as a function of time for the initial shape and the optimal shape, using the Newtonian constitutive equation for case 1. 

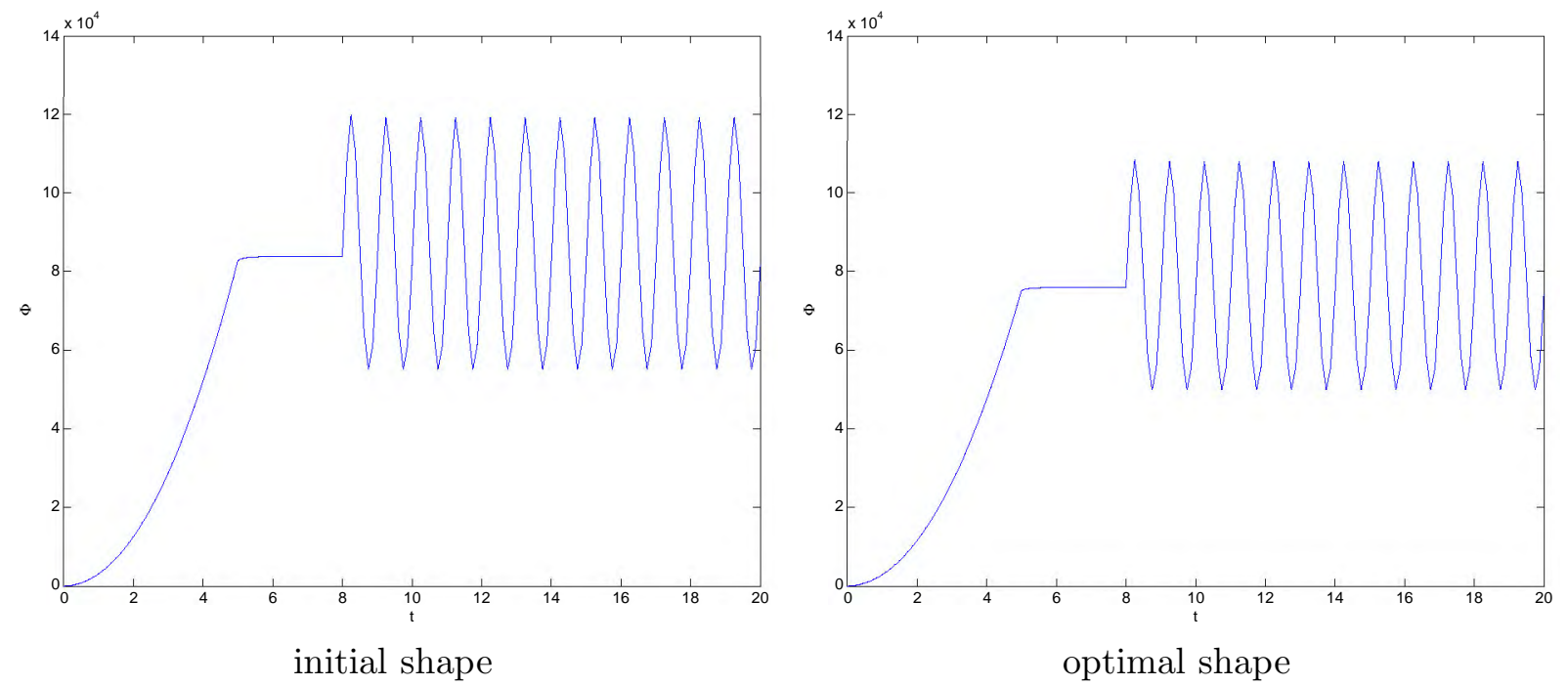

Figure 3. The function $\Phi\left(\mathbf{u}^{h}\right)$ as a function of time for the initial shape and the optimal shape, using the Carreau-Yasuda constitutive equation for case 1.

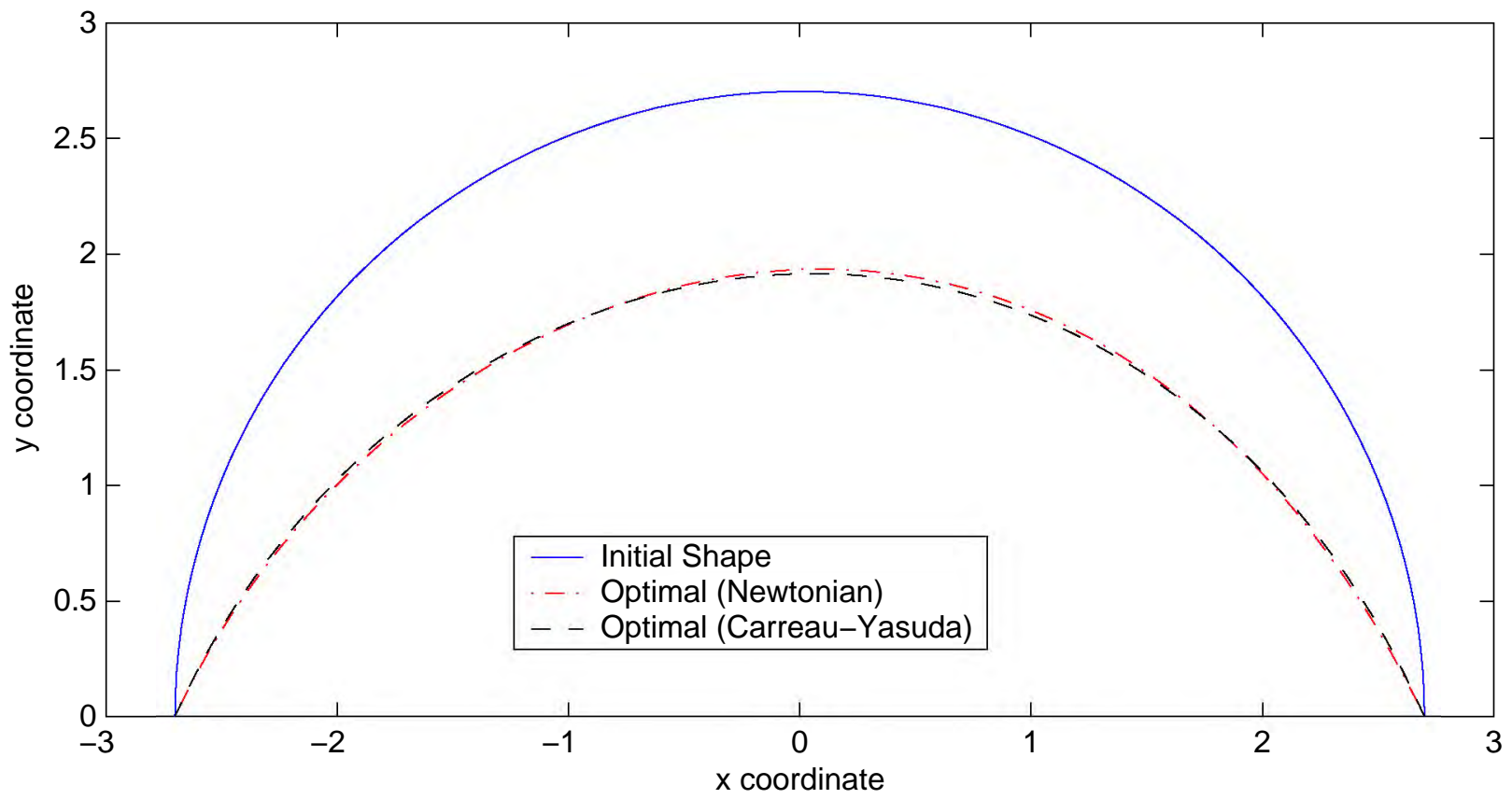

Figure 4. Initial and optimal shapes of the graft design curve for the Newtonian model for case 1. 


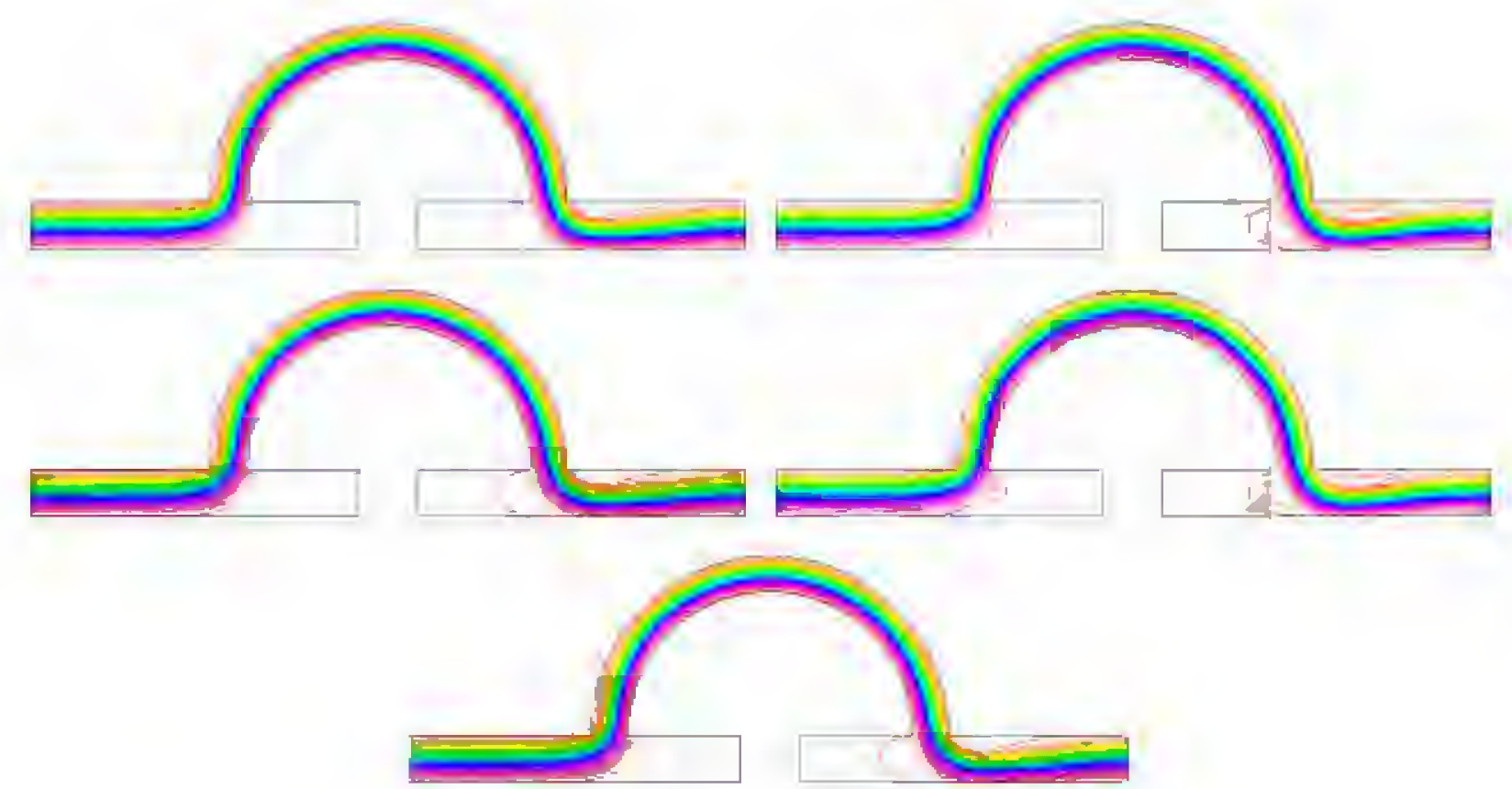

Figure 5. Streamline profiles at $t=15.0,15.25,15.50,15.75$ and 16.0, (left to right, then top to bottom) for the initial shape using Newtonian constitutive equations, for case 1.

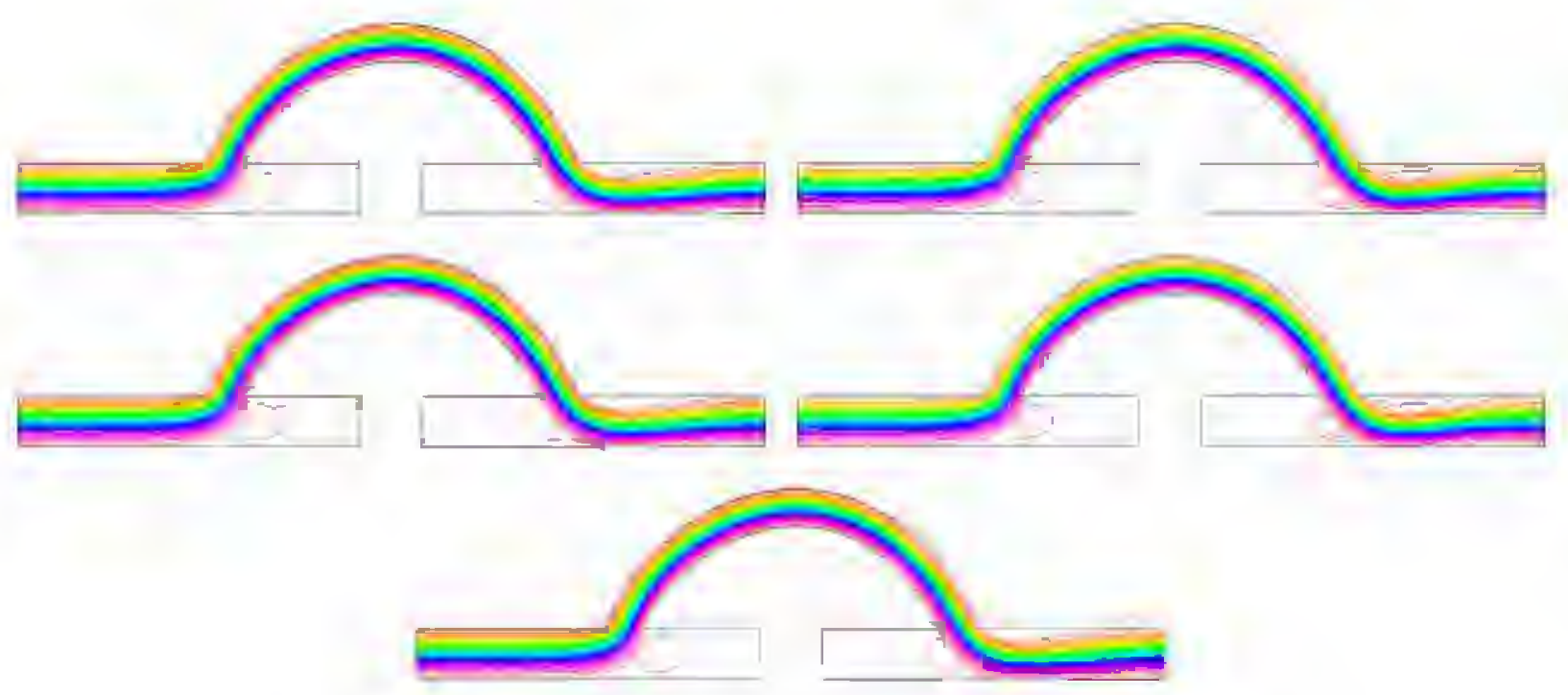

Figure 6. Streamline profiles at $t=15.0,15.25,15.50,15.75$ and 16.0, (left to right, then top to bottom) for the optimal shape using Newtonian constitutive equations, for case 1. 


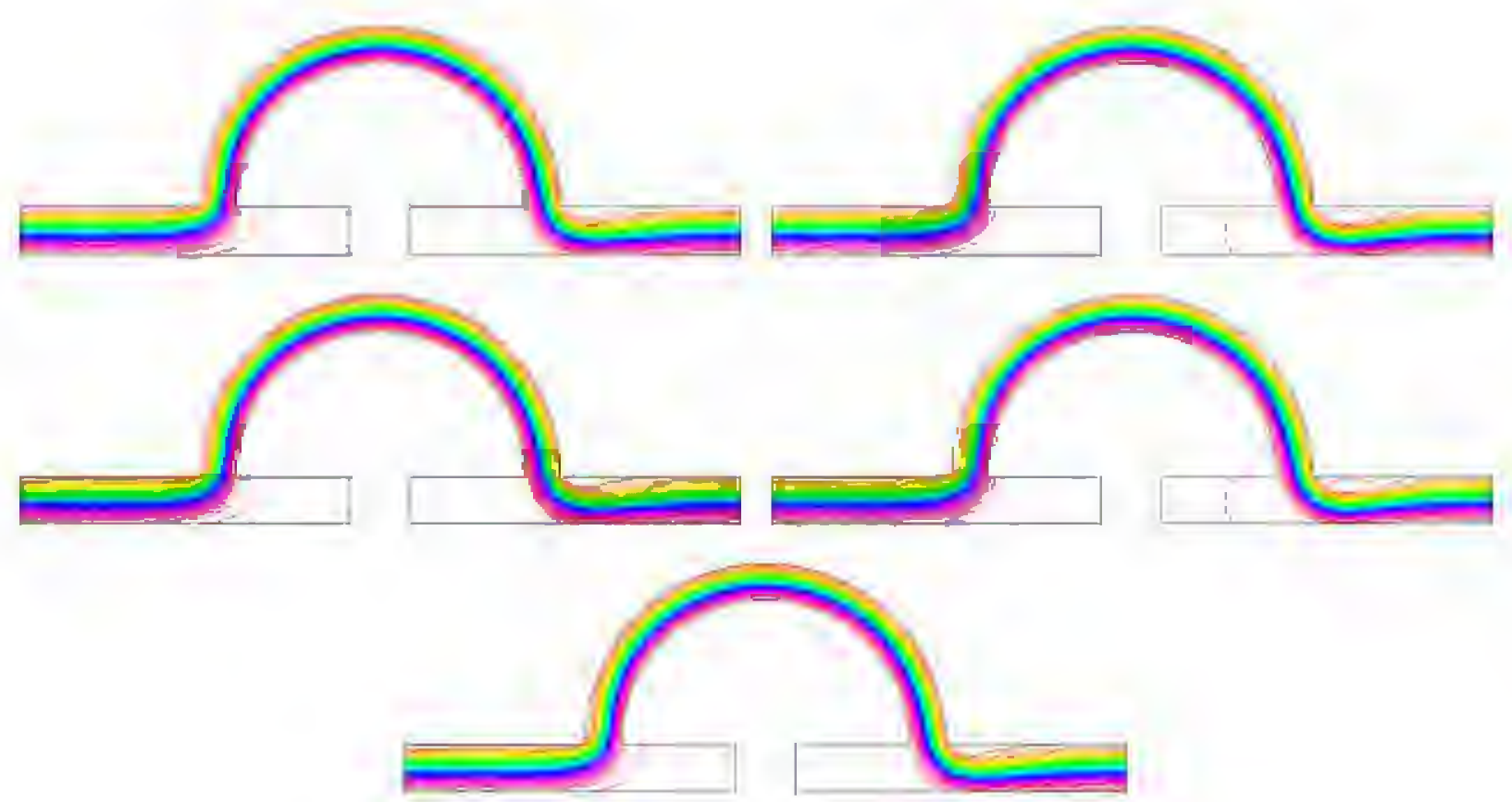

Figure 7. Streamline profiles at $t=15.0,15.25,15.50,15.75$ and 16.0, (left to right, then top to bottom) for the initial shape using Carreau-Yasuda constitutive equations, for case 1.

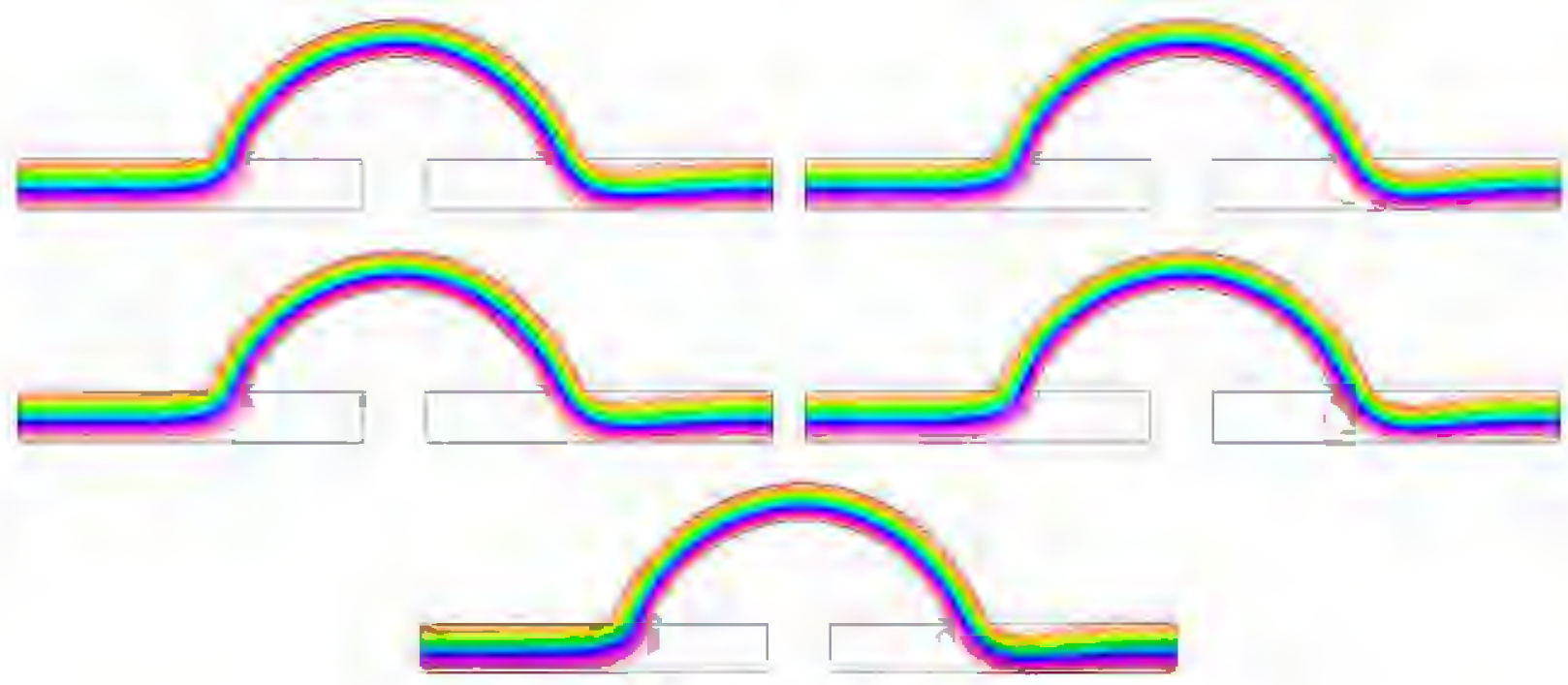

Figure 8. Streamline profiles at $t=15.0,15.25,15.50,15.75$ and 16.0, (left to right, then top to bottom) for the optimal shape using Carreau-Yasuda constitutive equations, for case 1. 


\subsection{Case 2}

The second case involves $d=1.0$, resulting in an aspect ratio of 1.25. The optimization process leads to a $16.57 \%$ reduction in the dissipation function using the Newtonian constitutive equation, with a gradient norm of $\left\|\nabla \widehat{J}\left(\boldsymbol{\alpha}_{0}\right)\right\| \times 10^{-7}$, and a $9.62 \%$ reduction in the dissipation function using the Carreau-Yasuda model, with a gradient norm of $\left\|\nabla \widehat{J}\left(\boldsymbol{\alpha}_{0}\right)\right\| \times 10^{-4}$. Figures 9 and 10 show the variation of $\Phi\left(\mathbf{u}^{h}\right)$ as a function of time for the initial and optimal shapes using the Newtonian and Carreau-Yasuda constitutive equations, respectively. Table 3 shows the computed optimal shape parameters defined in (20), and Table 4 shows the values of the angles of the graft, (from horizontal) at the inlet and exit of the artery. Figure 11 illustrates that the optimal shapes obtained using the Newtonian and Carreau-Yasuda constitutive equation choice do show a significant difference. Figures 12 and 13 show the streamline plots for the initial and optimal shapes, respectively, using the Newtonian constitutive equation within one period of the pulsatile phase of the flow. Figures 14 and 15 show the streamline plots for the initial and optimal shapes, respectively, using the CarreauYasuda constitutive equation within one period of the pulsatile phase of the flow.

\begin{tabular}{lrrr}
\hline \hline Design Variable & Initial & Newtonian & Carreau-Yasuda \\
\hline$r_{2}$ & 0.0 & 1.4809 & 1.3285 \\
$r_{3}$ & 0.0 & -0.7403 & -0.7090 \\
$r_{4}$ & 0.0 & 0.1714 & 0.1928 \\
$r_{5}$ & 0.0 & -0.0108 & -0.0177 \\
\hline \hline
\end{tabular}

Table 3. The design variables for the graft optimization for case 2 .

\begin{tabular}{ccrr}
\hline \hline Angle & Initial & Newtonian & Carreau-Yasuda \\
\hline$\theta=0$ & $90.00^{\circ}$ & $57.27^{\circ}$ & $60.18^{\circ}$ \\
$\theta=\pi$ & $90.00^{\circ}$ & $54.70^{\circ}$ & $63.84^{\circ}$ \\
\hline \hline
\end{tabular}

Table 4 . The angle that the graft makes with the artery at its inlet and exit, for case 2 .
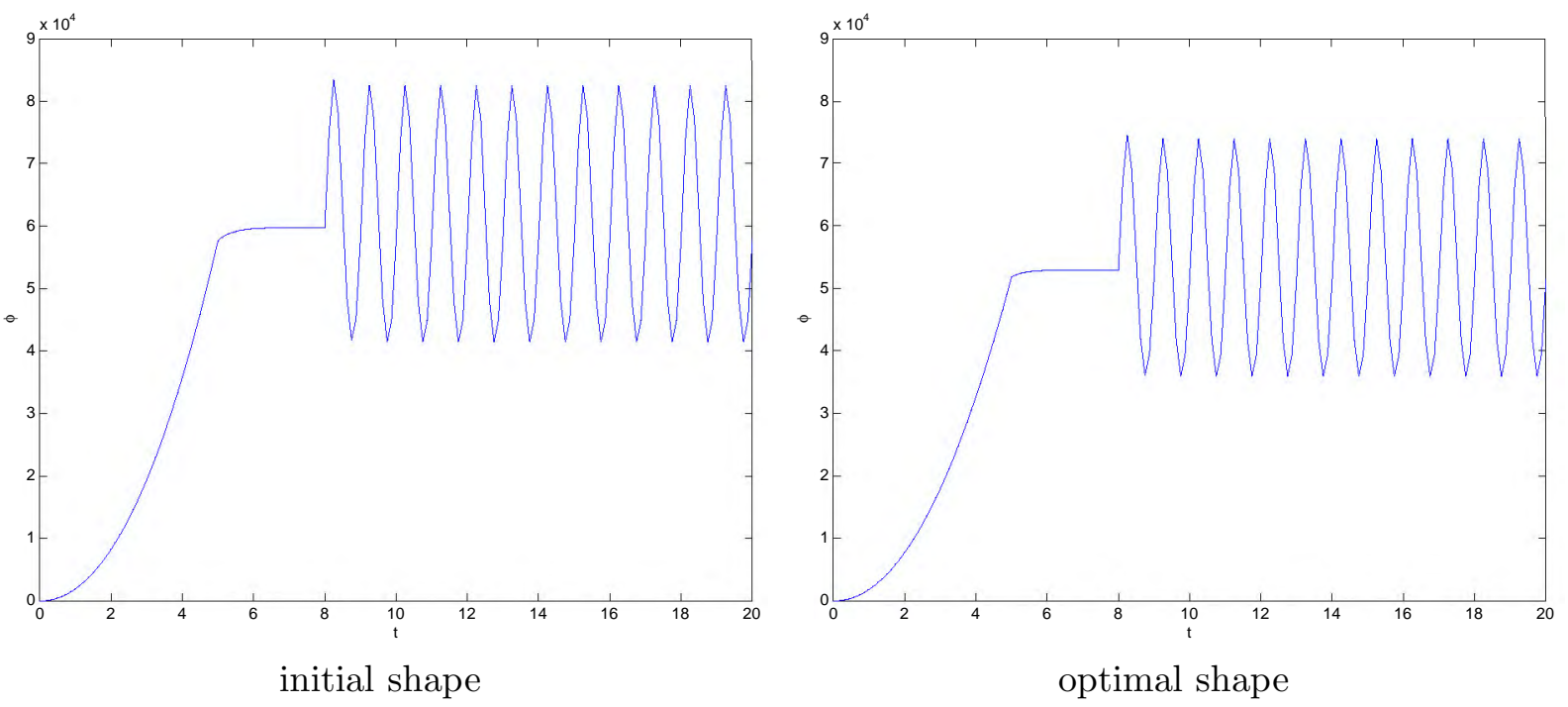

Figure 9. The function $\Phi\left(\mathbf{u}^{h}\right)$ as a function of time for the initial shape and the optimal shape, using the Newtonian constitutive equation for case 2 . 

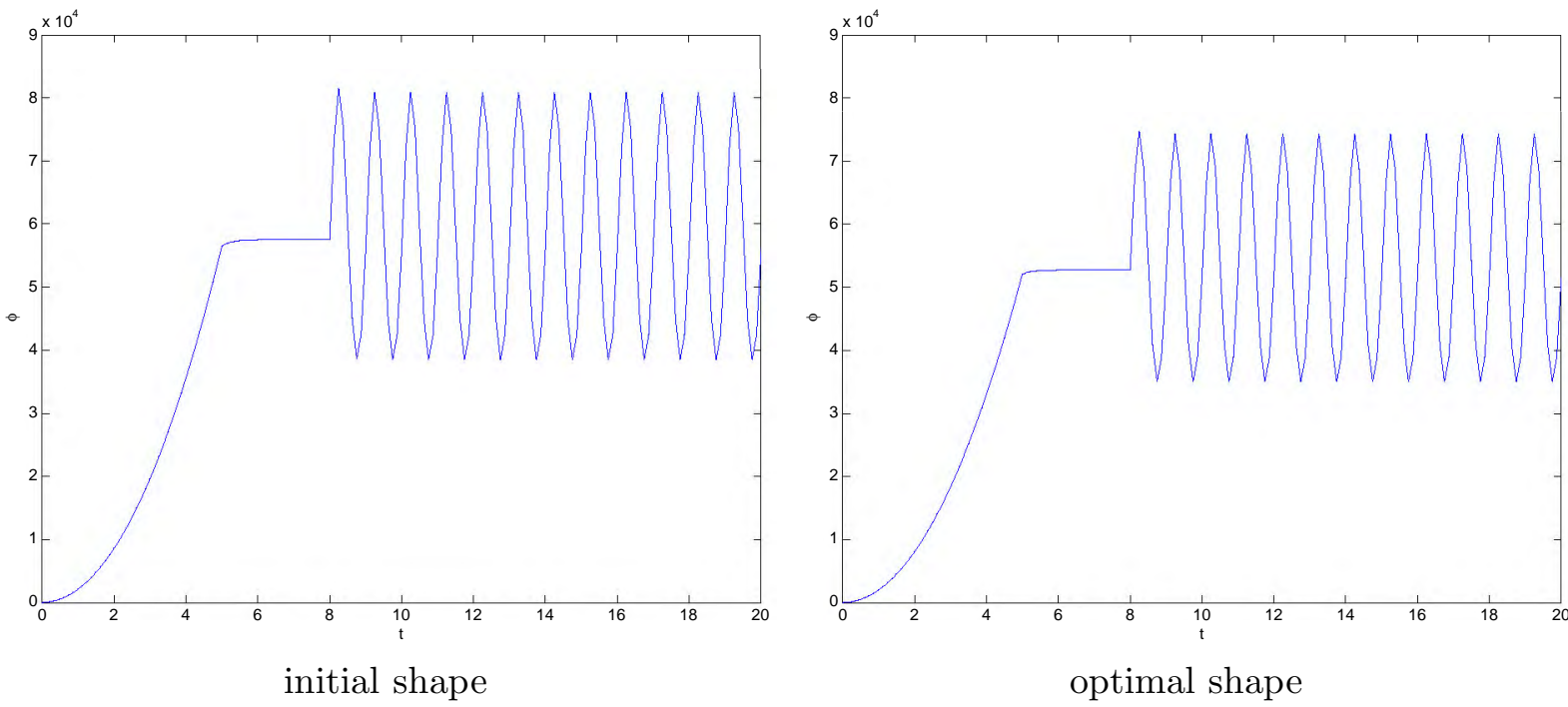

Figure 10. The function $\Phi\left(\mathbf{u}^{h}\right)$ as a function of time for the initial shape and the optimal shape, using the Carreau-Yasuda constitutive equation for case 2.

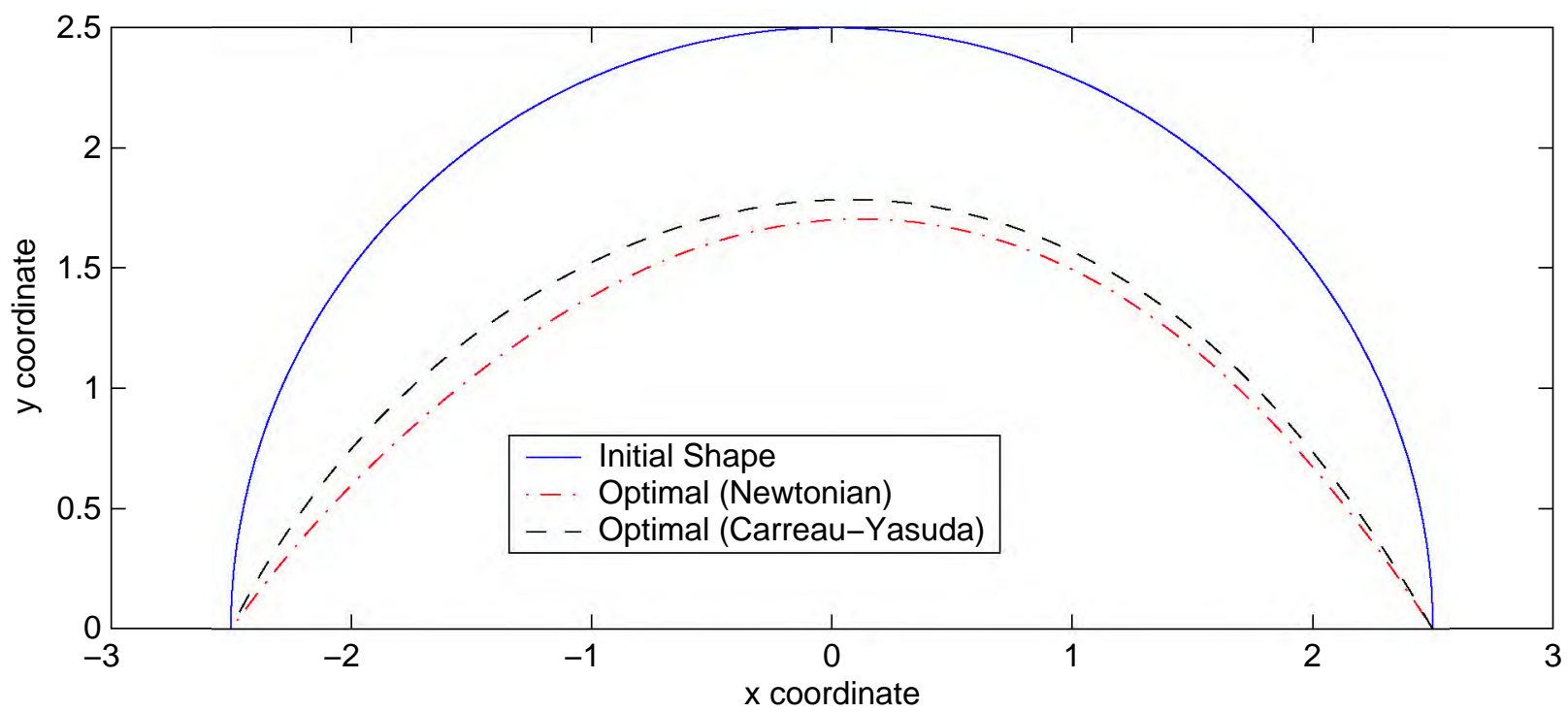

Figure 11. Initial and optimal shapes of the graft design curve for the Newtonian model for case 2.

\section{Conclusions}

We outlined the solution of shape optimization problems arising in the context of blood flow. The Carreau-Yasuda model was employed to account for the shear-thinning behavior of blood. The optimization problem is solved using a gradient-based method.

We considered an unsteady flow shape optimization problem for an idealized arterial graft geometry, with blood as the fluid. We see that, similarly to our results for the steady case [4], the thickness of the graft influenced the differences in the optimal shape obtained with respect to the constitutive equation choice. From (3), we see that a Newtonian assumption is valid for flows where shear rates are high. By increasing the aspect ratio, we are reducing the shear rate in the vicinity of the graft, and thereby increasing the shear-related differences. For the geometry with lower aspect ratio, i.e., case 1, we see that the differences between the flow field computed 


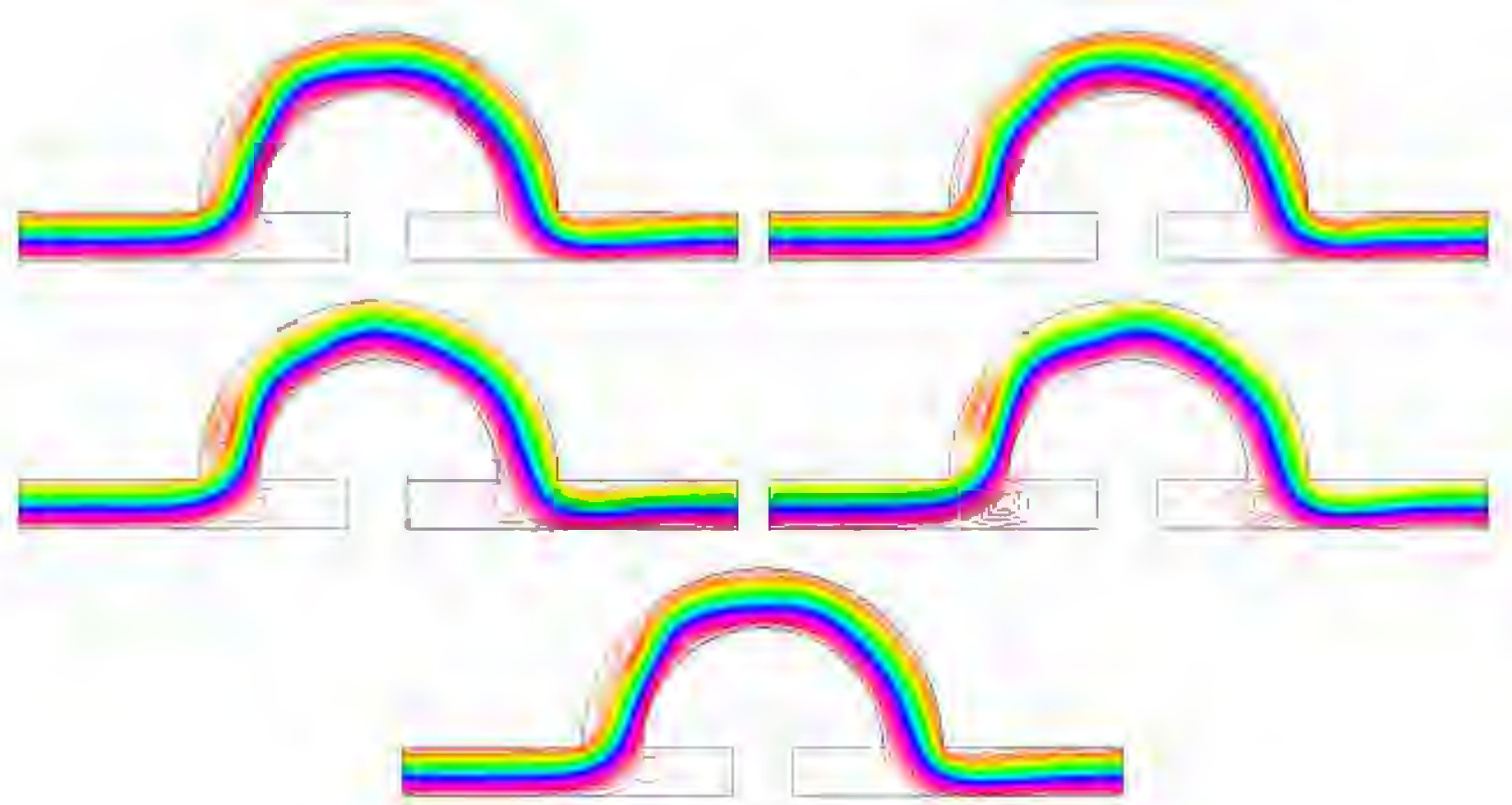

Figure 12. Streamline profiles at $t=15.0,15.25,15.50,15.75$ and 16.0, (left to right, then top to bottom) for the initial shape using Newtonian constitutive equations, for case 2 .

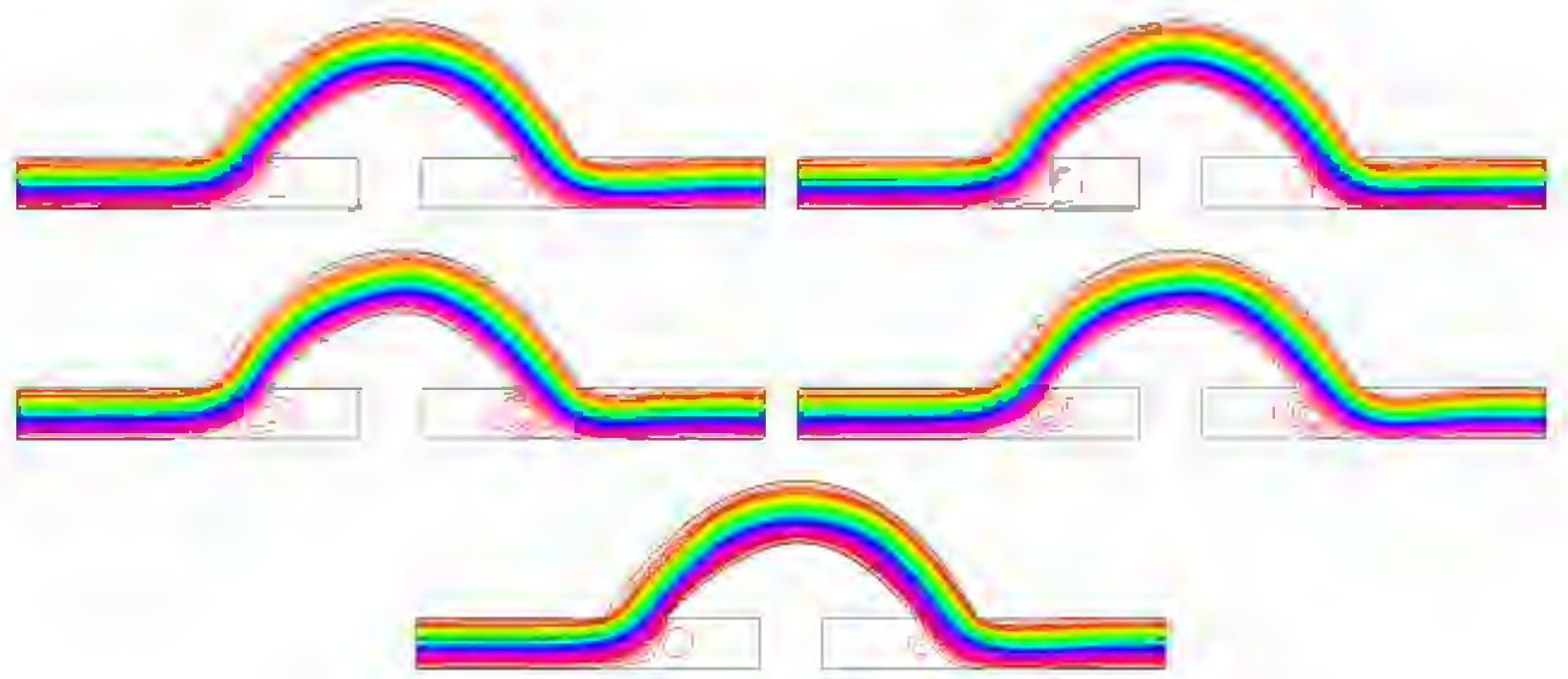

Figure 13. Streamline profiles at $t=15.0,15.25,15.50,15.75$ and 16.0, (left to right, then top to bottom) for the optimal shape using Newtonian constitutive equations, for case 2 .

using the Newtonian and generalized-Newtonian models are similar, for both the initial and final shapes, respectively. On comparing the flow field between the initial and optimal shapes, using either constitutive equation, we see that optimal shape design aims at reducing the recirculation region primarily in the vicinity of the entry and exit of the graft into the artery. We see that the optimal shapes for this case are almost identical (see Figure 4 and Table 1). For case 2, we see from Figure 12 and Figure 14, that there exists a striking difference in the flow field for the initial 


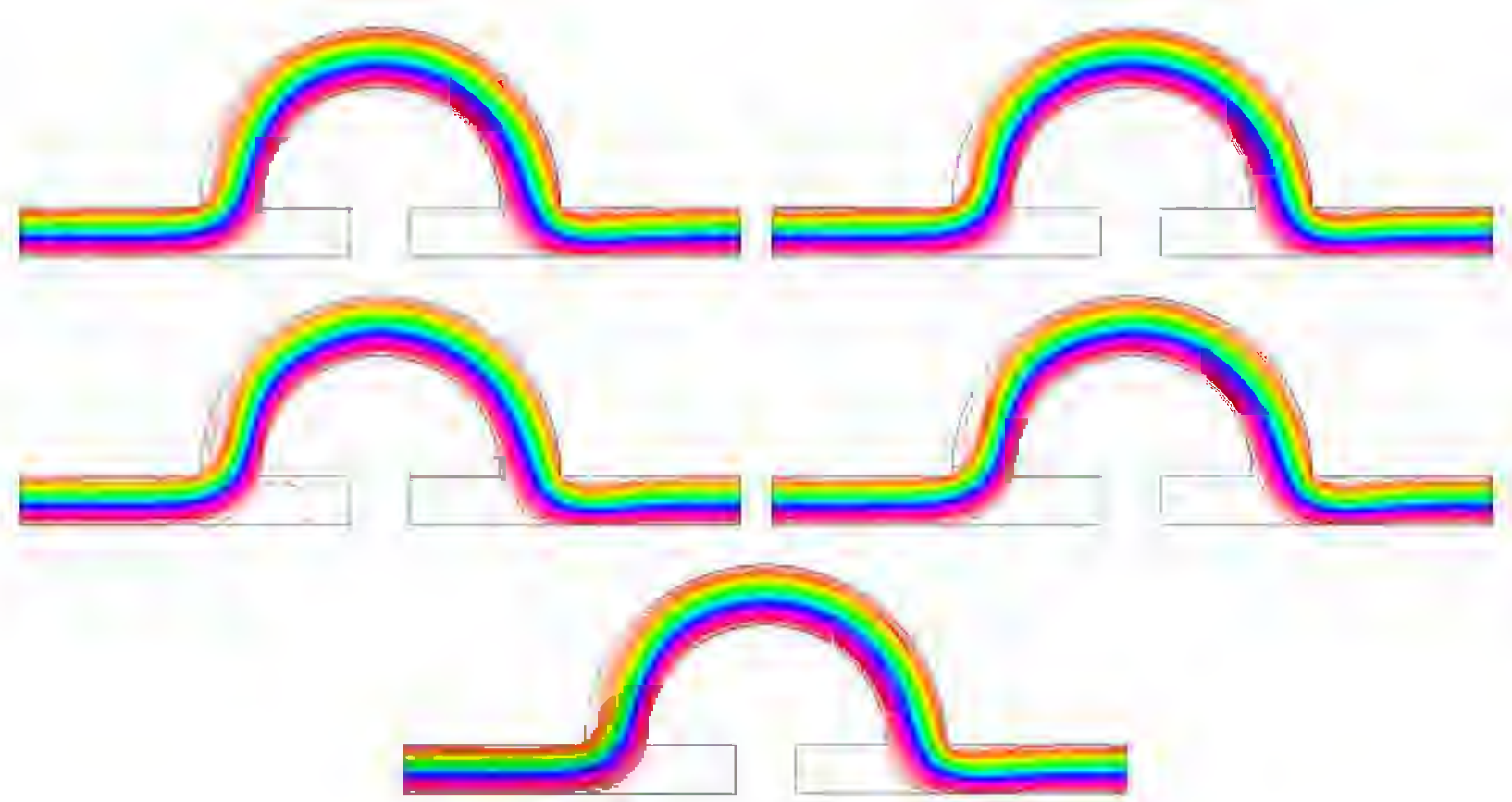

Figure 14. Streamline profiles at $t=15.0,15.25,15.50,15.75$ and 16.0, (left to right, then top to bottom) for the initial shape using Carreau-Yasuda constitutive equations, for case 2 .

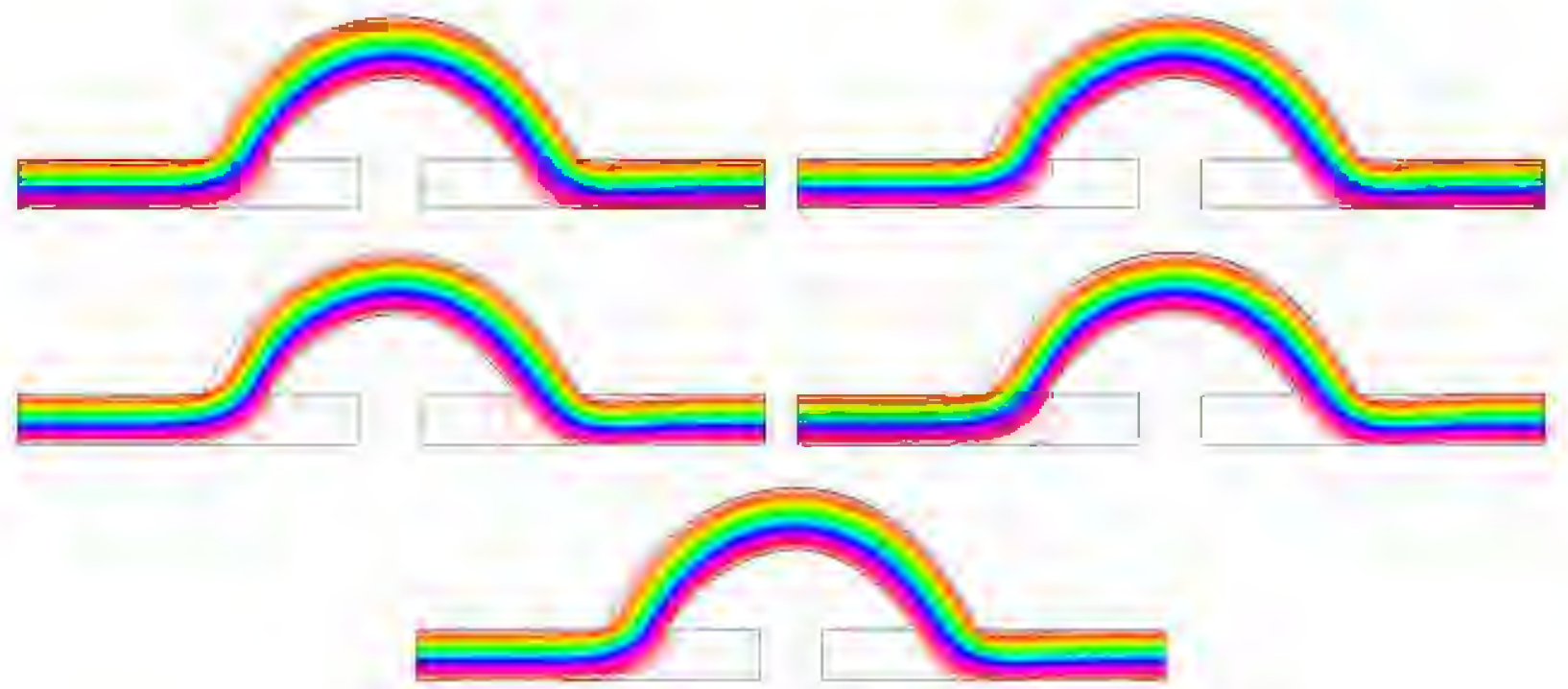

Figure 15. Streamline profiles at $t=15.0,15.25,15.50,15.75$ and 16.0, (left to right, then top to bottom) for the optimal shape using Carreau-Yasuda constitutive equations, for case 2 .

shape when comparing the Newtonian constitutive equation choice with that of the generalized Newtonian one, with the Newtonian model exhibiting a stronger recirculation within the graft. This accounts for the comparatively larger reduction in the objective function for the Newtonian choice, for this case. We also observe that there is a larger difference with respect to the choice of the constitutive model, in the angle formed between the graft and the artery at the inlet and exit 
(see Table 4). This difference is more pronounced at the graft inlet $(\theta=\pi)$, and may be attributed to the more violent flow field near this region when using the Newtonian model (see Figure 12).

Therefore, just like for the steady case, we conclude that when considering shape optimization problems involving blood, the shear rate in the domain affects the differences in optimal shapes obtained by the Newtonian and generalized Newtonian constitutive equations. For the test case we considered, these differences might not bear much engineering significance. However, for problems involving more complicated geometries, some of the geometric features therein might influence the outcome to a larger extent. In the future, we will be working on solving shape optimization problems for the design of the centrifugal blood pump.

\section{References}

[1] J.F. Antaki, O. Ghattas, G.W. Burgreen, and B. He, "Computational flow optimization of rotary blood pump components", Artificial Organs, 19 (1995) 608-615.

[2] G.W. Burgreen, J.F. Antaki, Z.J. Wu, and A.J. Holmes, "Computational fluid dynamics as a development tool for rotary blood pumps", Artificial Organs, 25 (2001) 336-340.

[3] A. Quarteroni and G. Rozza, "Optimal control and shape optimization in aorto-coronaric bypass anastomoses", Mathematical Models and Methods in Applied Sciences (M3AS), 13 (2003) 1801-1823.

[4] F. Abraham, M. Behr, and M. Heinkenschloss, "Shape optimization in stationary blood flow: A numerical study of non-Newtonian effects", submitted to Computer Methods in Biomechanics and Biomedical Engineering, 2004.

[5] A. Quarteroni, M. Tuveri, and A. Veneziani, "Computational vascular fluid dynamics: Problems, models and methods", Computing and Visualization in Science, 2 (2000) 163-197.

[6] F.J.H. Gijsen, F.N. van de Vosse, and J.D. Janssen, "The influence of the non-newtonian properties of blood on the flow in large arteries: Steady flow in a carotid bifurcation model", Journal of Biomechanics, 32 (1999) 601-608.

[7] P. Neofytou and D. Drikakis, "Effects of blood models on flows through a stenosis", International Journal for Numerical Methods in Fluids, 43 (2003) 597-635.

[8] B. He, O. Ghattas, and J.F. Antaki, "Computational strategies for shape optimization of Navier-Stokes flows", Technical Report CMU-CML-97-102, Computational Mechanics Lab, Department of Civil and Environmental Engineering, Carnegie Mellon University, 1997.

[9] M. Behr, D. Arora, and S. Schulte-Eistrup, "Prediction of flow features in centrifugal blood pumps", in Proceedings of the 2nd European Conference on Computational Mechanics (CDROM), Cracow University of Technology, Cracow, Poland, (2001).

[10] T.E. Tezduyar, M. Behr, and J. Liou, "A new strategy for finite element computations involving moving boundaries and interfaces - the deforming-spatial-domain/space-time procedure: I. The concept and the preliminary tests", Computer Methods in Applied Mechanics and Engineering, 94 (1992) 339-351.

[11] T.E. Tezduyar, M. Behr, S. Mittal, and J. Liou, "A new strategy for finite element computations involving moving boundaries and interfaces - the deforming-spatial-domain/space-time 
procedure: II. Computation of free-surface flows, two-liquid flows, and flows with drifting cylinders", Computer Methods in Applied Mechanics and Engineering, 94 (1992) 353-371.

[12] M. Behr and T.E. Tezduyar, "Shear-Slip Mesh Update Method", Computer Methods in Applied Mechanics and Engineering, 174 (1999) 261-274.

[13] M. Behr and D. Arora, "Shear-slip mesh update method: Implementation and applications", Computer Methods in Biomechanics and Biomedical Engineering, 6 (2003) 113-123.

[14] A. Leuprecht and K. Perktold, "Computer simulation of non-newtonian effects on blood flow in large arteries", Computer Methods in Biomechanics and Biomedical Engineering, 4 (2001) 149163.

[15] V. Girault and P. A. Raviart, Finite Element Methods for the Navier-Stokes Equations. Springer-Verlag, New York, 1986.

[16] M. D. Gunzburger, Finite Element Methods for Incompressible Viscous Flows: A Guide to Theory, Practice and Algorithms. Academic Press, Boston, 1989.

[17] D. Arora, M. Behr, and M. Pasquali, "A tensor-based measure for estimating blood damage", accepted to Artificial Organs, 2004.

[18] T.J.R. Hughes, L.P. Franca, and G.M. Hulbert, "A new finite element formulation for computational fluid dynamics: VIII. the Galerkin/least-squares method for advective-diffusive equations", Computer Methods in Applied Mechanics and Engineering, 73 (1989) 173-189.

[19] F. Shakib, Finite Element Analysis of the Compressible Euler and Navier-Stokes Equations, Ph.D. thesis, Stanford University, Department of Mechanical Engineering, 1988.

[20] F. Abraham, M. Behr, and M. Heinkenschloss, "The effect of stabilization in finite element methods for the optimal boundary control of the Oseen equations", Finite Elements in Analysis and Design, xx (2004) to appear.

[21] K. Jansen, S. S. Collis, C. Whiting, and F. Shakib, "A better consistency for low-order stabilized finite element methods", Comput. Methods Appl. Mech. Engrg., 174 (1999) 153-170.

[22] F. Abraham, Stabilized Finite Element Solution of Optimal Control Problems in Computational Fluid Dynamics, Ph.D. thesis, Rice University, Department of Mechanical Engineering and Materials Science, 2004.

[23] C. T. Kelley, Iterative Methods for Linear and Nonlinear Equations. SIAM, Philadelphia, 1995.

[24] B. He, O. Ghattas, and J. F. Antaki, "Computational strategies for shape optimization of Navier-Stokes flows", Technical Report CMU-CML-97-102, Computational Mechanics Lab, Department of Civil and Environmental Engineering, Carnegie Mellon University, 1997, http://www.cs.cmu.edu/ oghattas.

[25] J Haslinger and P. Neittaanmäki, Finite Element Approximation for Optimal Shape, Material, and Topology Design. John Wiley \& Sons, Chchester, New York, second edition, 1996.

[26] D. Ku, "Blood flow in arteries", Annual Review of Fluid Mechanics, 29 (1997) 399-434.

[27] J. Ryval, A.G.. Straatman, and D.A. Steinman, "Low Reynolds number modeling of pulsatile flow in moderately stenosed arteries", in 11th Annual Conference of the CFD Society of Canada, volume 1, Vancouver, Canada, (2003) 300-304. 